\title{
Forensic analysis of digital dynamic signatures: New methods for data
}

\section{treatment and feature evaluation}

Jacques Linden, MSc; Raymond Marquis, Ph.D. ; Williams Mazzella, Ph.D.

School of Criminal Justice, Lausanne, Switzerland.

ABSTRACT: The present study explored digital dynamic signatures containing quantifiable dynamic data. The change in data content and nature necessitates the development of new data treatment approaches. A SignPad Omega digitizing tablet was used to assess measurement reproducibility, as well as within writer variation and the frequency of correctly imitated features. Measurement reproducibility was found to be high except for pressure information. Within writer variation was found to be higher between days than on a same day. Occurrence of correct imitation was low for features such as signature size, trajectory length and total signature time. Feature discrimination factors combining within writer variability and the occurrence of correctly imitated features were computed and show that signature size, trajectory length and signature time are the features that perform the best for discriminating genuine from imitated signatures. A final experiment indicates that dynamic information can be used to create connections between imitation cases.

KEYWORDS: Forensic Science, Forensic Document Examination, Dynamic Signature, Data Analysis Methods, Variation study, Feature Discrimination

\section{Introduction}

Recently, forensic document examiners have been confronted with electronic signatures, also known as dynamic signatures. These handwritten signatures are electronically captured, often using a digitizing tablet, and include not only spatial but also temporal information. The first cases of fraud and dispute of such signatures are now appearing in Europe. Forensic scientists have started to study this field and the features of said signatures in detail. Authors such as Franke, Flynn, Nicolaides and Linton (1-4) have been active in exploring the dynamic characteristics and dynamic signatures in general. Few authors describe how they propose to deal with the data saved by a digitizing tablet, except using dedicated software developed for more general purposes. Harralson mentions a methodology for dynamic signatures, but does not give a practical example of the applied process (5). Thus, data extraction and treatment for dynamic signature remain vague and merit more attention from the scientific community. The present study was designed to explore and describe specific techniques to analyze the data extracted from a digitizing tablet. This includes already largely used 
global characteristics, which are individual measurements on a signature, and local characteristics, which are measured once per data point and constitute a more voluminous body of data.

The study treats four main aspects. First, it evaluates the reproducibility of measurements done by the digitizing tablet. Second, it shows different ways of describing and measuring within writer variation effectively, while discussing which alternative yields the best results. Third, it measures the frequency of correctly imitated features on a population of imitators. Finally, the within writer variation and comparison data was used to generate feature discrimination factors. These factors help recommending robust and useful features for discriminationv of genuine and questioned dynamic signatures.

\section{Materials and Methods}

\section{Materials}

A signotec SignPad Omega ${ }^{1}$ linked to a laptop computer was used to acquire the data. The tablet itself measures the $X$ and $Y$ coordinates of the input, the cumulated time associated for each coordinate couple and the pressure ( $Z$ coordinate). The temporal capture frequency of the digitizing tablet can be adjusted and was set at $500 \mathrm{~Hz}$ for this study. All signatures were written using the plastic pen provided with the digitizing tablet.

As a first step, feature reproducibility was tested using a "SignaScript Atlantic+" auto-pen device (7).

\section{Participants}

This study focused on three low complexity signatures (Figure 1). These signatures are abbreviated signatures using only the individual's initials. The main reasons for this choice were the fact that they are easy to learn and imitate, and further constitute difficult cases encountered by question document examiners. They present little information compared to more complex signatures. They are also easy to analyze and provide a smaller set of variables for a pilot research.

Three students at the School of Criminal Justice, all in their twenties, were asked to provide thirty signatures each. Ten signatures were provided on three different days, in order to gather data on the subject's internal variation. The three types of signatures are illustrated below.

\footnotetext{
${ }^{1}$ 6. Signotec. Signotec Homepage. 2016 [updated 2016; cited 2016 20.01.2016]; Available from: http://www.signotec.com/produkte/unterschriften-pads/omega-pad/.
} 


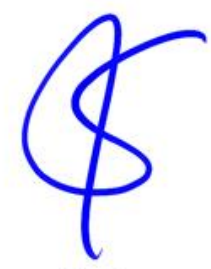

(a)

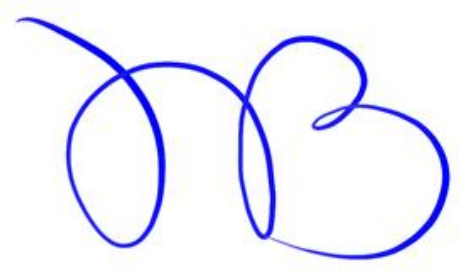

(b)

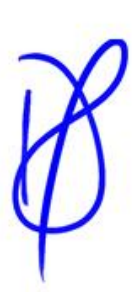

(c)

Figure 1 - Genuine signatures (a), (b) and (c)

Signature (a) is constituted of a single stroke forming the letters " $\mathrm{S}$ " and " $\mathrm{K}$ ". Both are melded into a single compact sign. Signature (b) consists of a single stroke. The signature is formed by the letters " $\mathrm{L}$ " and "B" written in a slow, arcing manner. The letters are easily recognizable and bear a lot of resemblance to cursive writing. Signature (c) consists of two strokes forming the letters " $\mathrm{D}$ " and " $\mathrm{f}$ ". The form is reminiscent of a " $G$ " key in musical notation. Both letters are intertwined, but remain easily recognizable.

Five scanned samples of signatures (a), (b) and (c) were sent to twenty students and collaborators of the School of Criminal Justice. They were asked to train and imitate the three types of signatures and were allowed to familiarize themselves with the digitizing tablet beforehand. Every participant then provided three imitations for each of the reference signatures using the digitizing tablet. Afterwards, they were asked to select their subjectively best imitation after visual inspection of the digital ink trace.

\section{Features}

In the following paragraphs, the expressions "questioned signature" and "genuine signature" will be used. By this, the authors mean signatures whose sources are unknown, in contrast to genuine signatures which come from a known source.

All the signatures were embedded into a pdf file using the manufacturer's SignoSign/2 software. The signatures were then extracted and decrypted using the Signotec RSAVerifier. The raw $\operatorname{csv}^{2}$ files were further imported into the R software package (8). All data treatments and measurements were done on the measurements from the tablet, not on inked traces or images of the signatures.

\section{Global Features (Table 1 \& Table 2)}

Global characteristics are features where one single measurement (or statement if the feature is nonquantifiable and subjectively assessed) is extracted per signature, based on all of the available data points (9).

\footnotetext{
${ }^{2}$ CSV - Comma Separated Values
} 


\begin{tabular}{|c|c|c|}
\hline FEATURE & TYPE & DESCRIPTION \\
\hline SHAPE & Global & Subjective assessment of shape \\
\hline LINE QUALITY & Global & Fluent (undented) or not fluent (dented) line \\
\hline SLOPE & Global & $\begin{array}{l}\text { Subjective assessment of signature slope (steep left, left, straight, } \\
\text { right, steep right) }\end{array}$ \\
\hline $\begin{array}{l}\text { BOUNDING BOX } \\
\text { DIMENSIONS }\end{array}$ & Global & $\begin{array}{l}\text { Measurements of the bounding box surrounding all the signature } \\
\text { (in } \mathrm{cm} \text { ) }\end{array}$ \\
\hline BOX RATIO & Global & Bounding box height divided by its width \\
\hline $\begin{array}{l}\text { TRAJECTORY } \\
\text { LENGTH }\end{array}$ & Global & $\begin{array}{l}\text { Length of the trajectory as described by the tip of the pen } \\
\text { (excluding pen lifts) (in } \mathrm{cm} \text { ) }\end{array}$ \\
\hline PARTICULARITIES & Global & $\begin{array}{l}\text { Includes elements such as proportions within the signature, letter } \\
\text { proportions, initial stroke direction }\end{array}$ \\
\hline \multicolumn{3}{|c|}{ Table 1 - Static ${ }^{3}$ feature description } \\
\hline FEATURE & TYPE & DESCRIPTION \\
\hline $\begin{array}{l}\text { SIGNATURE } \\
\text { TIME }\end{array}$ & Global & Time needed to write the whole signature, including pen lifts \\
\hline PEN-UP TIME & Global & $\begin{array}{l}\text { Duration of each pen lift (corresponding to the repositioning of the } \\
\text { pen) }\end{array}$ \\
\hline STOPS & Global & $\begin{array}{l}\text { Number of stops (without lifting the pen) in the process that are } \\
\text { longer than } 35 \mathrm{~ms} \text { (11) }\end{array}$ \\
\hline EXECUTION & Global & $\begin{array}{l}\text { Elements of how the signature was executed, including direction, } \\
\text { starting point and ending point, as well as stroke sequence }\end{array}$ \\
\hline PRESSURE & Local & $\begin{array}{l}\text { Pressure value, measured in levels defined by the digitizing tablet } \\
\text { (on a scale from } 0 \text { to 1023) }\end{array}$ \\
\hline VELOCITY & Local & Tangential velocity, calculated between data points (in $p x / s)^{4}$ \\
\hline ACCELERATION & Local & $\begin{array}{l}\text { Tangential acceleration, calculated between every velocity value } \\
\text { (in } p x / s^{2} \text { ) }\end{array}$ \\
\hline
\end{tabular}

Table 2 - Dynamic ${ }^{5}$ feature description

\footnotetext{
${ }^{3}$ « Handwriting or signature written with pen on paper. [...] Some electronically captured signatures may be analyzed through their graphic, static forms without the aid of temporal data. " 10. Harralson HH. Glossary. In: Harralson HH, editor. Developments in Handwriting and Signature Identification in the Digital Age: Anderson Publishing, Ltd.; 2013;125-8.

${ }^{4}$ Picture cells (pixels) per second.

${ }^{5}$ « Refers to electronically captured temporal handwriting features such as velocity, pressure, and duration. The dynamics of the signature refer to the movement patterns of the handwriting. " 10. Harralson $\mathrm{HH}$.
} 


\section{Local Features (Table 2)}

Local features were extracted for each sample point in the input domain (9), thus they generally formed a large body of data with each measurement being associated to a precise region on the signature. As each measurement has a precise context and space on the signature trajectory, "repositioning" the values graphically was essential so that the local features could be properly understood and assessed. The large data volume complicates comparison and creates the need for visualization techniques and mathematical comparison tools, such as Dynamic Time Warping (DTW) ${ }^{6}$.

\section{Segmental Features}

A third kind of features were measured, segmental features, where the signature is subdivided into segments (9). These features can be obtained by grouping the local data by a criterion of choice, the inflection of trajectory direction in this case.

\section{Reproducibility}

Measurement reproducibility was assessed using the autopen device on five different regions of the digitizing tablet (the four corners and the middle) and with three different pressure settings (high, medium and $\left(0 \mathrm{w}^{7}\right) . \mathrm{X}$ - and $\mathrm{Y}$-Coordinates, pressure and time were compared qualitatively within and between regions. The auto-pen was chosen to eliminate natural variation in these trials. A total of five signatures per region and pressure were created using the autopen, making 75 signatures in total.

Glossary. In: Harralson HH, editor. Developments in Handwriting and Signature Identification in the Digital Age: Anderson Publishing, Ltd.; 2013;125-8.

${ }^{6}$ " Used in online signature recognition. The algorithm measures similarity between two sequences when there are variances in time between the sequences. [...] Time is warped in one signature so that two recorded handwriting patterns optimally resemble each other, both in space and time. » $10 . \quad$ Ibid.

${ }^{7}$ Adjusted with a screw on the autopen machine. 


\section{Comparison scheme}

Comparisons between reference sets and questioned materials were done using boxplots of the reference values (thirty signatures total from the three different days) and comparing the questioned value to the reference boxplot. The value measured from the questioned signature was then positioned as being in the main variation (box), the rim regions (upper and lower 25\%) or as an outlier. All cases that were on the rim of the variation were classified as accepted, only outliers were classified as refused. This categorical statement scheme yielded high acceptance rates and may produce false positives from the rim values that may cause underestimation of feature utility.

Genuine signatures were grouped together (thirty signatures per writer) and compared to each other to generate within writer variation. For local features, day-to-day comparison was carried out by DTW. The sets per day, formed of ten signatures, were taken one by one and each signature in a set was compared to each signature in a second set. The mean distance from those comparisons was then plotted as a boxplot to illustrate day-to-day variation. As for genuine and imitation comparison, each feature was compared to the writer's standard value and classified as inside or outside natural variation, yielding a frequency per feature.

The imitated signatures were also compared to the set of genuine signatures. Each feature was measured against the genuine set and the feature value was either classified as different or not matching. This yielded an occurrence of correctly imitated features.

\section{Within writer variation and occurrence of correctly imitated features}

The second step was the assessment of genuine signatures variation for each of the chosen samples. Within writer variation was studied with signatures taken on three different days. Variation was thus assessed within a single day as well as between days. Occurrence of correctly imitated features was studied using a database of the twenty best imitations per reference signature $(a, b$ and $c)$.

For the global features we chose boxplots to visualize within writer variation measurements. As such values could be plotted in a set containing all genuine signature values and a point containing the value measured on a questioned signature, as can be seen in Figure 2 below. Features were classified as either being in- or outside of the total observed variation. This comparison scheme was applied for all quantifiable characteristics. Non-quantifiable characteristics were subjectively qualified and it was then determined whether or not they matched. 

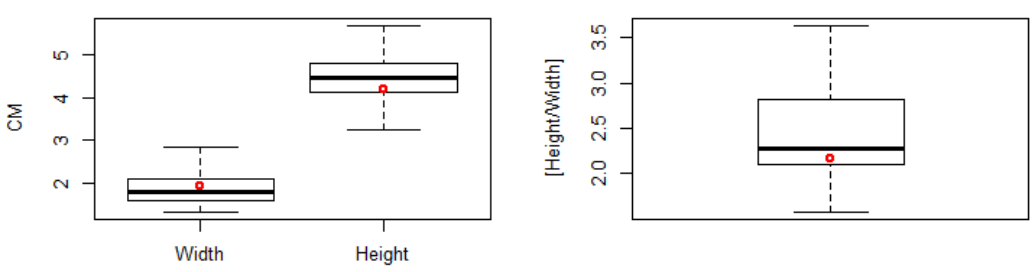

Proportion

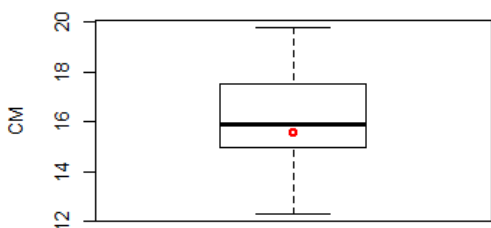

Trajectory length

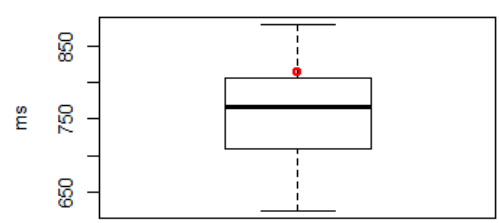

Total time

Figure 2 - Illustration of the model used to assess whether features can be differentiated or not. Boxplots represent measurements for the thirty genuine signatures. The red point represents the measurement of the questioned signature.

For local characteristics, the three following approaches were applied:

The first approach consisted in a qualitative comparison by either plotting multiple feature curves (generally two) on the same graph (superposition) or by aligning several graphs (juxtaposition). The plotted curves were the characteristics intensity as a function of the timing or advancement $\left(\frac{\text { Actual time }}{\text { Total signature time }} \times 100\right)$. The main criteria for comparison were the general curve profile (general trends in the curve), the position of the main peaks as well as their intensity, as can be seen in Figure 3. 

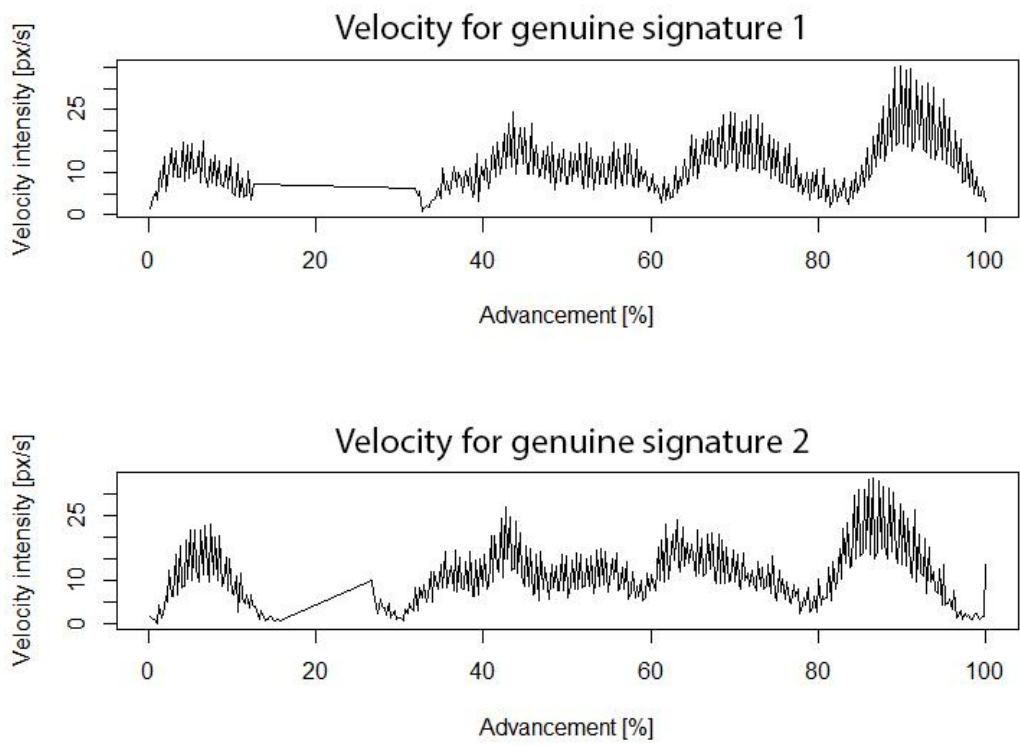

Figure 3 -Example of a feature graph comparison by juxtaposition of two genuine signature (c)

The second approach uses a Dynamic Time Warping (DTW) algorithm, created by Giorgino (12) for the $\mathrm{R}$ software package, to mathematically compare the local characteristics. A symmetrical DTW approach allowing only for complete graph compression/extension was chosen in order to keep the computation times low. The dissimilarity between curves was measured using Euclidean distance. As for the reference signatures, the data from the thirty genuine signatures were compared for each combination, creating a large body of Euclidean distance data. All distances, 435 for a thirty signature set, were then used to create a boxplot to quantify within writer variation for the local features. Questioned signatures were compared to each signature in the reference set (thirty comparisons in total for a given questioned signature) and the mean distance was plotted with the reference set's distance boxplot. Comparison was carried out in the same way as for the global characteristics, where the mean distance between the questioned signature data and the reference set was used to determine whether or not the values were in- or outside of the genuine writer's variation.

The third and last approach consisted in segmenting the signature into smaller units in order to extract movement related information. Signatures were analyzed for changes in direction (horizontal and vertical) that, by hypothesis, impose movement constraints on the signatory. As an example, a sharp turn would impose a deceleration of the pen and a decrease in pressure, while a large rounded turn would only require a lesser change in dynamics. Straight segments between separation points should show acceleration at the beginning, stable velocity in the middle and deceleration at the end. Segments between turning points were thus supposed to be more homogenous and comparable on a dynamic scale, as they constitute a dynamic unit. An example of the resulting segmentation is given in Figure 4 
and Figure 5. An algorithm detecting changes in direction was developed, additional or missing points were corrected manually to equalize the number of segments. For the reference set, each segment was grouped with its counterpart in the other signatures. Then boxplots for each dynamic characteristic were generated to show variation per segment and the stepwise rises and declines between segments. This data was then plotted against the mean values with the standard deviation for the questioned signature.

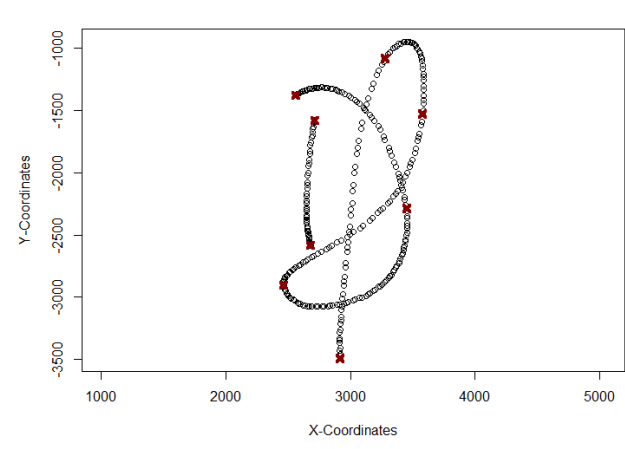

Figure 4 - Changes in trajectory direction in signature (c).

Each cross is a segmentation point determined automatically

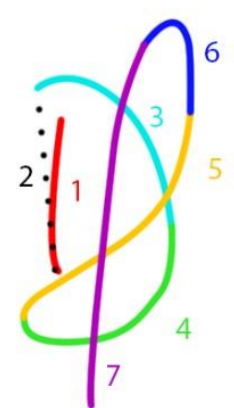

Figure 5 - Segments resulting from segmentation in signature (c)

\section{Feature discrimination factor}

The third step was to compute a feature discrimination factor, a concept similar to discriminative power. Occurrence of genuine signatures fitting into the normal variation of the genuine signatures for the given feature, was divided by the occurrence of imitated signatures fitting into the normal variation of the genuine signature. The ratio of these occurrences combines within writer variation and between writer variation data from a trained imitator population. The feature discrimination factor expresses whether or not that feature might be useful to classify a signature into either the genuine or into the imitated signature population. A high ratio means that the feature has high potential to differentiate both populations and thus is useful for the forensic document examiner, while values close to one imply low discrimination and impact on the classification. The estimated feature discrimination ratio is in no way an approach to evaluate evidence findings. The proposed approach is purely aimed at determining which feature is most useful for classification and should possibly be prioritized or more highly valued during the comparison phase. 


\section{Results and discussion}

\section{Reproducibility}

Results showed that reproducibility of graphical features is very high with a few minor deviations, as can be seen in Figure 6 .

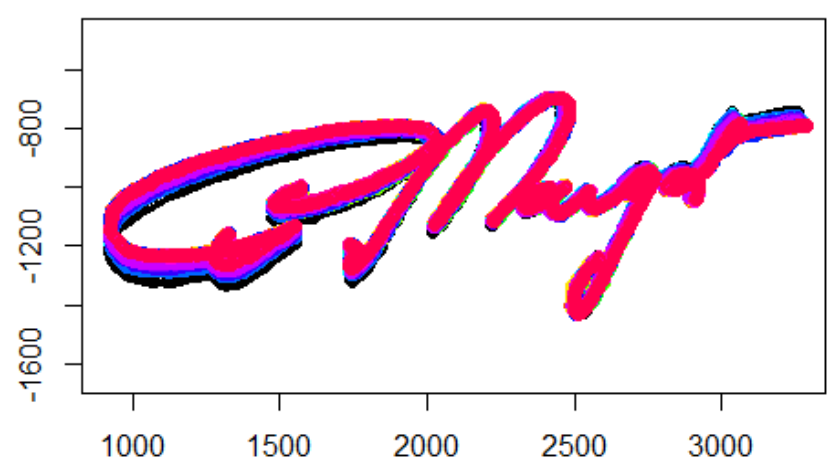

Figure 6 - Superimposition of twenty autopen signatures from different regions of the tablet and on different pressure settings

Twenty autopen signatures can be superimposed almost exactly, independently of the region where they were captured or the applied pressure. The few minor variations may be the result of the imperfect pen fixation to the autopen device. Total signature time varied very slightly. The highest variation was observed for the pressure information. The signal differed strongly between different regions on the digitizing tablet, making capture position an important factor. Results showed that intensity values on the low pressure settings were often higher or equivalent to the values obtained using higher pressure settings. This led to the hypothesis that a signal pre-treatment (either amplification or reduction based on signal strength) takes place for both low and high pressure signals, thus rendering the use of a mean pressure value as comparison feature useless. Authors report different conclusions on the use of pressure, some obtain better results $(1,13-15)$ while integrating pressure into their classification models and some others claim signification only if the signal matches (5). Because the pressure measurement was region dependent, it was considered non reliable and was discarded. This result highlights the need to check measurement reproducibility for each digitizing system.

\section{Dynamic data analysis approaches}

Three different techniques to visualize and compare the local dynamic information were applied to the data set.

The first technique involves simple visualization of the feature's data, followed by qualitative comparison. From a methodological standpoint this method has the advantage of taking into account 
all available information. The operator can detect small gaps in the graphs, corresponding to natural or unnatural stops in the execution of the signature. All this information can be used to infer and recreate the writing sequence and process, as well as detect signs of imitation. The biggest disadvantage of this method is its time consuming nature. An operator has to compare curves in small batches in order to see irregularities. Generating an intra-variation using this process takes a long time and necessitates focus and precise note taking. Another disadvantage is the "subjective" nature of the process. While the process is not arbitrary, it is still operator-dependent. Differences between operators, such as different measurement regions or even comparison methodology, may cause measurement incompatibility and differing interpretations of the information. Specific criteria, such as which peaks need to measured or discounted and where the measurement should take place on the peak, need to be fixed in order to reduce the impact of the operator's influence.

The second technique involves the automatic comparison of the time series using a symmetric dynamic time warping algorithm. Once the parameters are chosen, two data sets are inserted and the distance computed. Whole sets can be treated in a relatively short time, which is very useful for generating within writer variation distances. Questioned signatures are compared to the set using median and shortest distances to determine matches and mismatches. In this approach, the comparison is a "black box", making the comparison procedure less transparent. Thus, DTW does not inform the questioned document examiner on hesitations and stops in the execution, but it is an easy and fast method for comparing large amounts of dynamic data. It is also operator independent, which makes it a more objective way of qualifying (dis-) similarity of graphs. Quantitative results are more easily comprehensible and justifiable than the qualitative results produced with the first method. The local data often presents values that are situated in the rim regions of the genuine signatures' variation using this approach, which are cases where the correspondence can point either way, depending on the two distributions being compared. Being able to extract those distributions and combine them with the other (global and local) features is the first step to being able to form a coherent conclusion even in difficult cases.

The third technique involves signature segmentation by changes in trajectory direction. Signatures were segmented on specific points, separating each signature into several line segments. Signature (a) was segmented into six parts, signature (b) into eight parts and signature (c) into seven parts. The approach fell short of expectations. A few problems were inherent to the segmentation, as the irregularity and natural variation in the signature's execution characteristics limit the segmentation point count and position reproducibility. Another problem was the large size of the segments, which entailed high variation and further lowered the usefulness of the method for classification purposes. A lot of the imitated signature data superimposed with the genuine signature data, whereas the other 
methods illustrated clear differences between the data. Still, a segmentation approach may inform the questioned document examiner about the more detailed execution of the sub-movements involved in the creation of the signature. It also is useful for determining trends in the decomposed movements (Figure 7, Figure $8 \&$ Figure 9) and understanding the execution of the genuine signatures. The previously cited illustrations show median velocity or acceleration values for each signature segment, which can be seen in graphs such as Figure 10 and Figure 11. These median values show that the strokes have a consistent pattern (ups and downs between segments) and that this pattern is reproduced on several days. While not adapted for comparison purposes, the approach may yet have potential for research and variation studies.

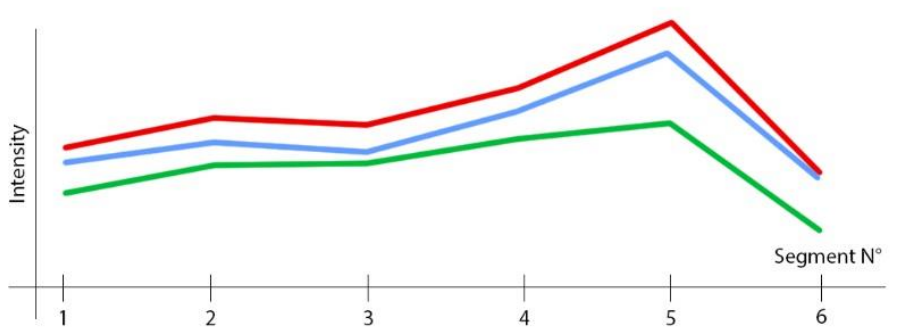

Figure 7 - Trend in median velocity in the signature segments of signature (a). Each colour corresponds to a day. Curves are staggered vertically for clarity.

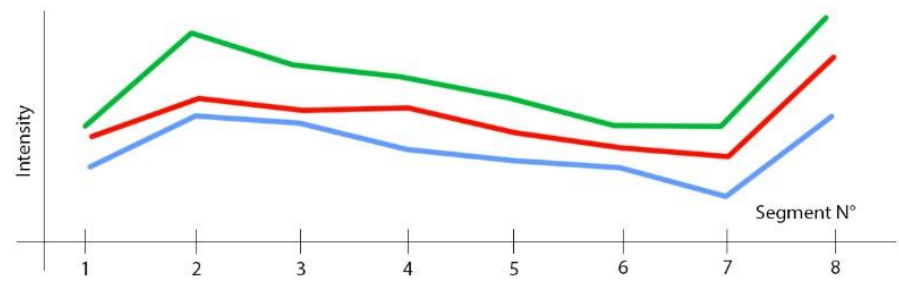

Figure 8 - Trend in median velocity in the signature segments of signature (b). Each colour corresponds to a day. Curves are staggered vertically for clarity.

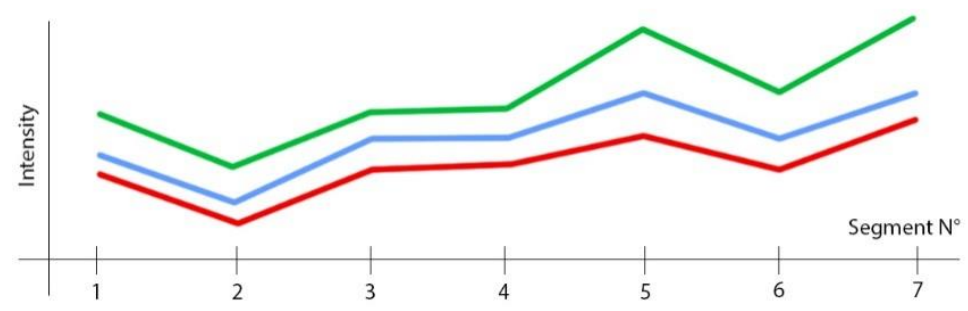

Figure 9 - Trend in median velocity in the signature segments of signature (c). Each colour corresponds to a day. Curves are staggered vertically for clarity. 


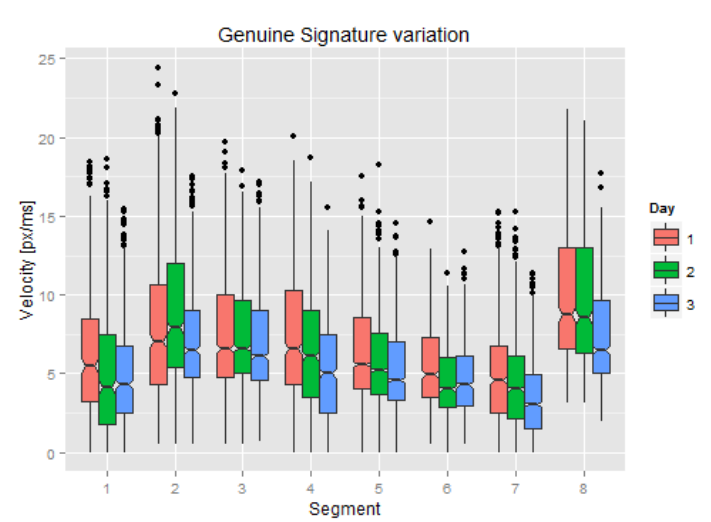

Figure 10 - Signature (b) variation for velocity

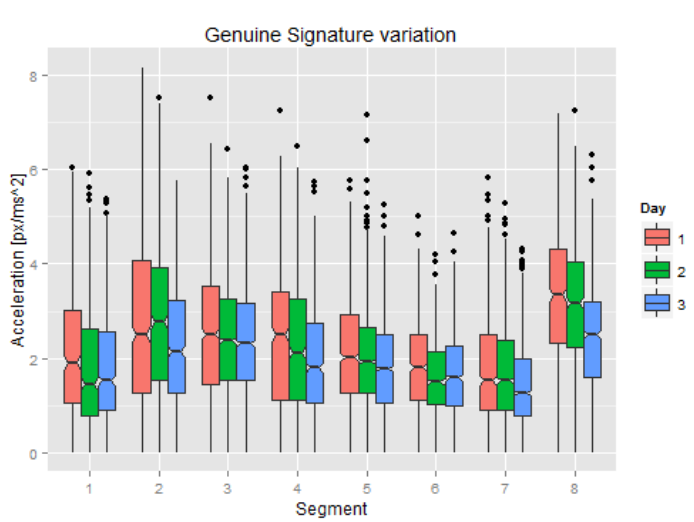

Figure 11 - Signature (b) variation for acceleration

\section{Within writer variation}

Variation was studied in detail for each of the three signatures. Qualitatively assessed features, (e.g., form, slope and line quality) were stable for each of the signatures. None of the thirty genuine signatures presented any outliers for these features. All of them displayed a good fit in terms of form and slope. All the signatures showed good, smooth and dynamic line quality. There were no significant differences observed on these macroscopic features acquired on signatures written on different days. The categories defined as particularities showed differing amount on variation depending on the characteristics. Some, such as stroke angle and presence of stroke end artifacts in the form of hooks were very variable, but this variation was global and independent of the day. Others, such as internal letter proportions and proportions between letters were more stable and reliable. Those features produced the same results for each signature. Only the particularities and specific features with low variation mentioned above were chosen from the pool of particularities for the genuine-imitation comparisons, the reason being the low chance of discrimination between the two.

The quantitatively assessed features produced more noticeable variation between signatures acquired on different days. Features such as width and height varied often on the scale of a few millimeters within a day (Figure 12 \& Figure 13). Height seems to be a more variable feature than width, two out of three writers had quite large variations within a single day. Between days, values were always at least partially superimposed, suggesting stability in signature size. Again, signature height seemed to be more variable between days than width. As for the bounding box ratio (

Figure 14), variation within days was often higher, while median values seem to be conserved even between days. Again, all of the values superimpose at least partially. Trajectory length showed large variations, on the scale of several centimeters (Figure 15). Variation within days was often quite large, but between days values superimpose partially. 
Total signature time (Figure 16 \& Figure 17) had complex variation and was quite signature dependent. Within days, values were often grouped quite closely (variation of about $100 \mathrm{~ms}$ ), but variation was often quite significant between days, having only very limited superimposition. The (b) signature was quite problematic concerning this feature, as signature time seemed to get slower every day. Still, signature time is undoubtedly linked to trajectory length, which is also higher for the samples taken on days two and three, possibly explaining some of the variation. Additionally this high variation might also be explained by the fact that signature (b) was only recently adopted by the signatory. The writer of signature (b) had to sign a lot of documents for her job and changed her signature only three months prior to the examination, possibly leaving her still in a learning phase, where features and execution stabilize. The third signature was the only with a pen-up movement, but the pen-lift duration was reproducibly short, both within and between days, as can be seen in Figure 18.

Dynamic feature variation was measured using the segmentation approach and the dynamic time warping method. The segmentation approach yielded the general trends for corresponding features, if one examined the median values for example. Repeatable patterns and trends were observed for velocity and acceleration. Even though variation is clearly present, general high and low regions as well as peaks are conserved between days. The DTW method clearly shows that in velocity (Figure 19) and acceleration (

Figure 20) graphs, within day distances are lower than between day distances.

Generally speaking, variation between days is higher than within day variation. This appears to be especially noticeable for dynamic features. This fact reaffirms the need for multiple samples taken on different days, as suggested by Thiéry (16).

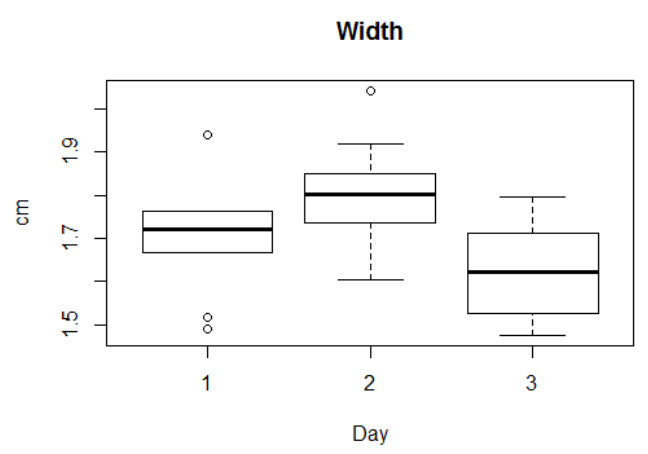

Figure 12 - Signature (a) variation for width

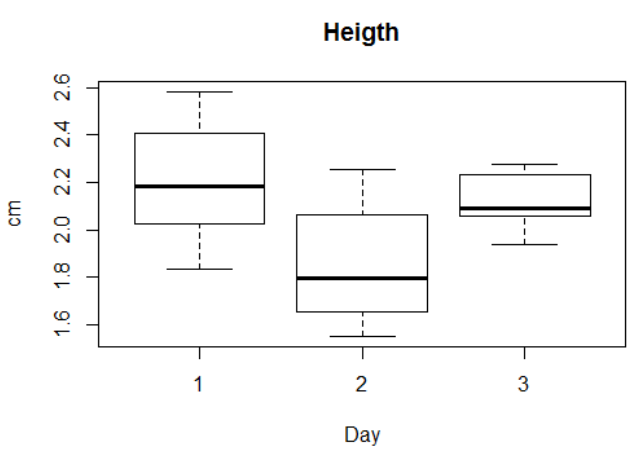

Figure 13 - Signature (a) variation for height 
Page $\mathbf{1 5}$ of $\mathbf{2 2}$

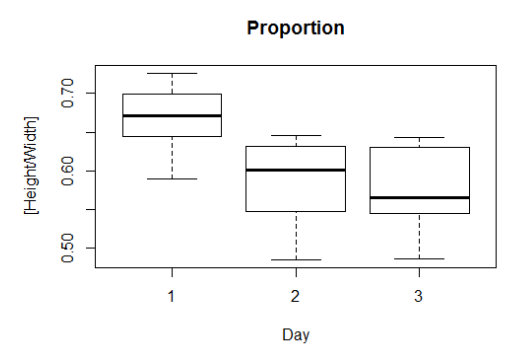

Figure 14 - Signature (b) variation for box ratio

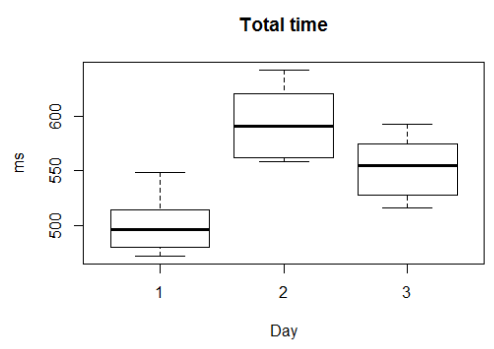

Figure 16 - Signature (a) variation for total signature time

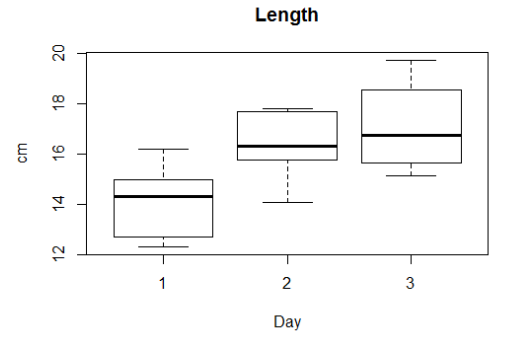

Figure 15 - Signature (c) variation for trajectory length

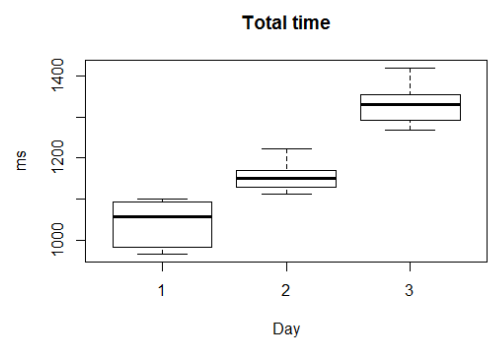

Figure 17 - Signature (b) variation for total signature time

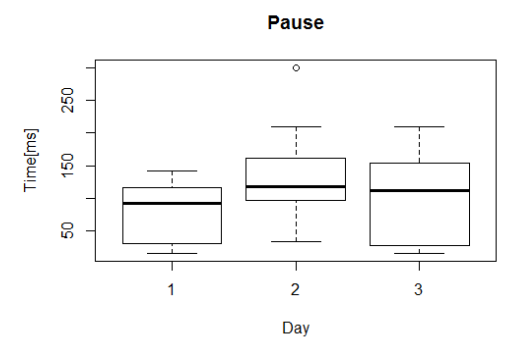

Figure 18 - Signature (c) variation for pen-up time

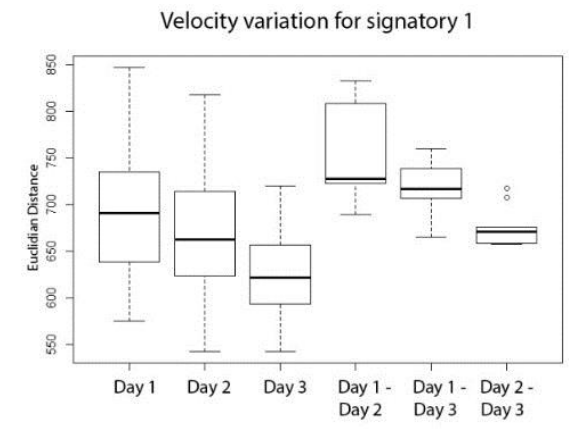

Figure 19 - Signature (a) within and between day distances for velocity

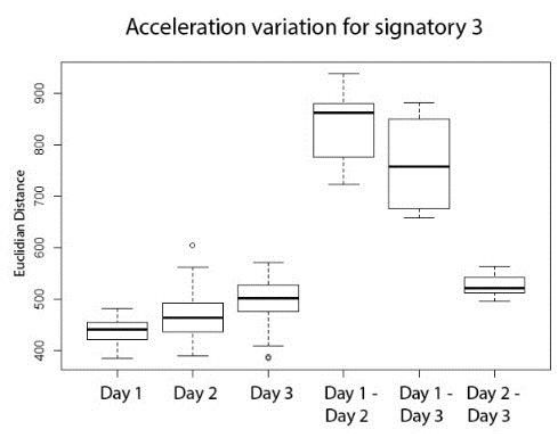

Figure 20 - Signature (c) within and between day distances for acceleration 
The chosen comparison approach yielded a very high "tolerance" for feature measurements and thus high acceptance rates. Specifically, the acceptance percentage for each static characteristic was $100 \%$, except for trajectory length, which had $97 \%$ acceptance for signature (a). As for the dynamic features, the same is true, except for velocity and acceleration in signature (a), which were at $98 \%$ acceptance and $99 \%$ for acceleration in signature (b).

\section{Occurrence of correctly imitated features}

As for the within writer variation, the comparison classification scheme into accepted and rejected values leads to high percentages detailed in Table 3 and Table 4.

\begin{tabular}{|c|c|c|c|c|c|c|}
\hline & \multicolumn{2}{|c|}{ SIGNATURE (A) } & \multicolumn{2}{|c|}{ SIGNATURE (B) } & \multicolumn{2}{|c|}{ SIGNATURE (C) } \\
\hline $\begin{array}{l}\text { CONCLUSION } \\
\text { FEATURE }\end{array}$ & Accepted & Rejected & Accepted & Rejected & Accepted & Rejected \\
\hline SIGNATURE SHAPE & $70 \%$ & $30 \%$ & $55 \%$ & $45 \%$ & $80 \%$ & $20 \%$ \\
\hline LINE QUALITY & $80 \%$ & $20 \%$ & $80 \%$ & $20 \%$ & $100 \%$ & $0 \%$ \\
\hline SIGNATURE SLOPE & $90 \%$ & $10 \%$ & $80 \%$ & $20 \%$ & $75 \%$ & $25 \%$ \\
\hline $\begin{array}{l}\text { BOUNDING BOX } \\
\text { DIMENSIONS }\end{array}$ & $40 \%$ & $60 \%$ & $40 \%$ & $60 \%$ & $15 \%$ & $85 \%$ \\
\hline BOUNDING BOX RATIO & $75 \%$ & $25 \%$ & $15 \%$ & $85 \%$ & $75 \%$ & $25 \%$ \\
\hline TRAJECTORY LENGTH & $35 \%$ & $65 \%$ & $25 \%$ & $75 \%$ & $15 \%$ & $85 \%$ \\
\hline PARTICULARITIES & $45 \%$ & $55 \%$ & $25 \%$ & $75 \%$ & $50 \%$ & $50 \%$ \\
\hline \multicolumn{7}{|c|}{ Table 3 - Static feature ratio of correctly imitated features } \\
\hline & \multicolumn{2}{|c|}{ SIGNATURE (A) } & \multicolumn{2}{|c|}{ SIGNATURE (B) } & \multicolumn{2}{|c|}{ SIGNATURE (C) } \\
\hline $\begin{array}{l}\text { CONCLUSION } \\
\text { FEATURE }\end{array}$ & Accepted & Rejected & Accepted & Rejected & Accepted & Rejected \\
\hline TOTAL SIGNATURE TIME & $35 \%$ & $65 \%$ & $45 \%$ & $55 \%$ & $15 \%$ & $85 \%$ \\
\hline STOPS & $100 \%$ & $0 \%$ & $85 \%$ & $15 \%$ & $100 \%$ & $0 \%$ \\
\hline PEN-UP TIME & - & - & - & - & $35 \%$ & $65 \%$ \\
\hline EXECUTION/SEQUENCE & $85 \%$ & $15 \%$ & $90 \%$ & $10 \%$ & $80 \%$ & $20 \%$ \\
\hline VELOCITY (DTW) & $75 \%$ & $25 \%$ & $60 \%$ & $40 \%$ & $40 \%$ & $60 \%$ \\
\hline ACCELERATION (DTW) & $70 \%$ & $30 \%$ & $60 \%$ & $40 \%$ & $70 \%$ & $30 \%$ \\
\hline
\end{tabular}

Table 4 - Dynamic feature ratio of correctly imitated features 


\section{Feature discrimination factors}

The data from the within writer variation and the imitation studies was then used to compute feature discrimination factors. Within writer variation was divided by the occurrence of correctly imitated features. The resulting ratios express a measure similar to discriminative power and are given in Table 5 and Table 6.

FEATURE

TRAJECTORY LENGTH
BOUNDING BOX DIMENSIONS
BOUNDING BOX RATIO
PARTICULARITIES
SIGNATURE SHAPE
SIGNATURE SLOPE
LINE QUALITY

SIGNATURE (A) SIGNATURE (B) SIGNATURE (C)

MEAN

$\begin{array}{llll}2.86 & 4.00 & 6.67 & 4.51 \\ 2.50 & 2.50 & 6.67 & 3.89 \\ 1.33 & 6.67 & 1.33 & 3.11 \\ 2.22 & 4.00 & 2.00 & 2.74 \\ 1.43 & 1.82 & 1.25 & 1.50 \\ 1.11 & 1.25 & 1.33 & 1.23 \\ 1.25 & 1.25 & 1.00 & 1.17\end{array}$

Table 5 - Static feature discrimination factors

\begin{tabular}{c|cccc} 
FEATURE & SIGNATURE (A) & SIGNATURE (B) & SIGNATURE (C) & MEAN \\
\hline TOTAL SIGNATURE TIME & 2.86 & 2.22 & 6.67 & 3.92 \\
PEN-LIFT DURATION & - & - & 2.86 & 2.86 \\
VELOCITY (DTW) & 1.31 & 1.66 & 2.50 & 1.82 \\
ACCELERATION (DTW) & 1.40 & 1.64 & 1.43 & 1.49 \\
DIRECTION / EXECUTION & 1.18 & 1.11 & 1.25 & 1.18 \\
STOPS & 1.00 & 1.18 & 1.00 & 1.06
\end{tabular}

Table 6 - Dynamic feature discrimination factors

\section{Feature discrimination factors}

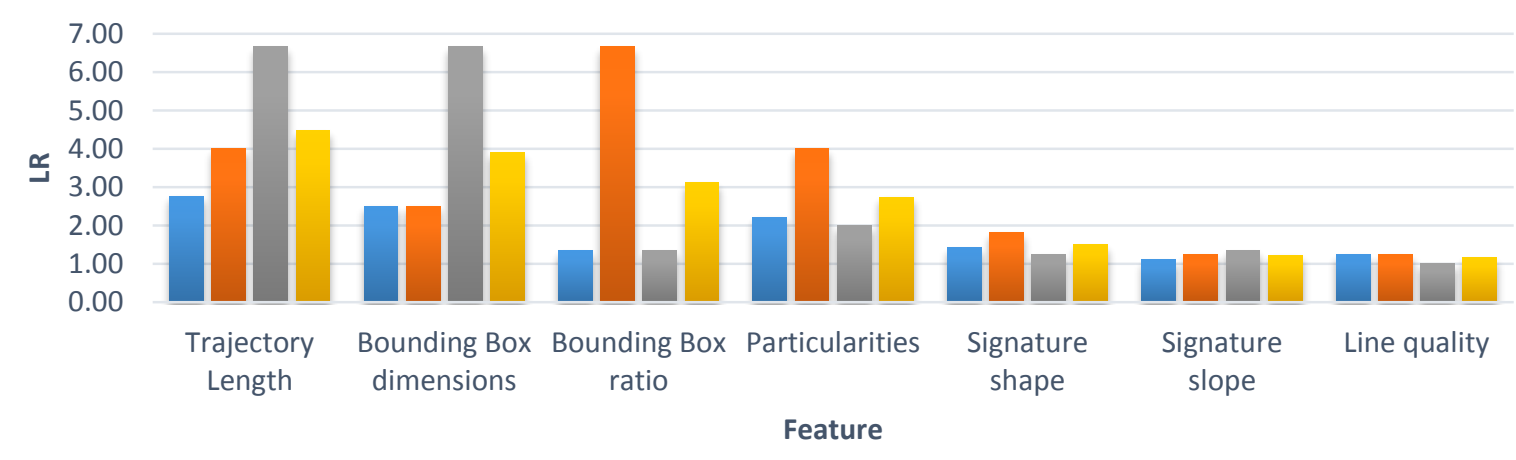

घignature 1 - Signature 2 Signature 3 Mean 


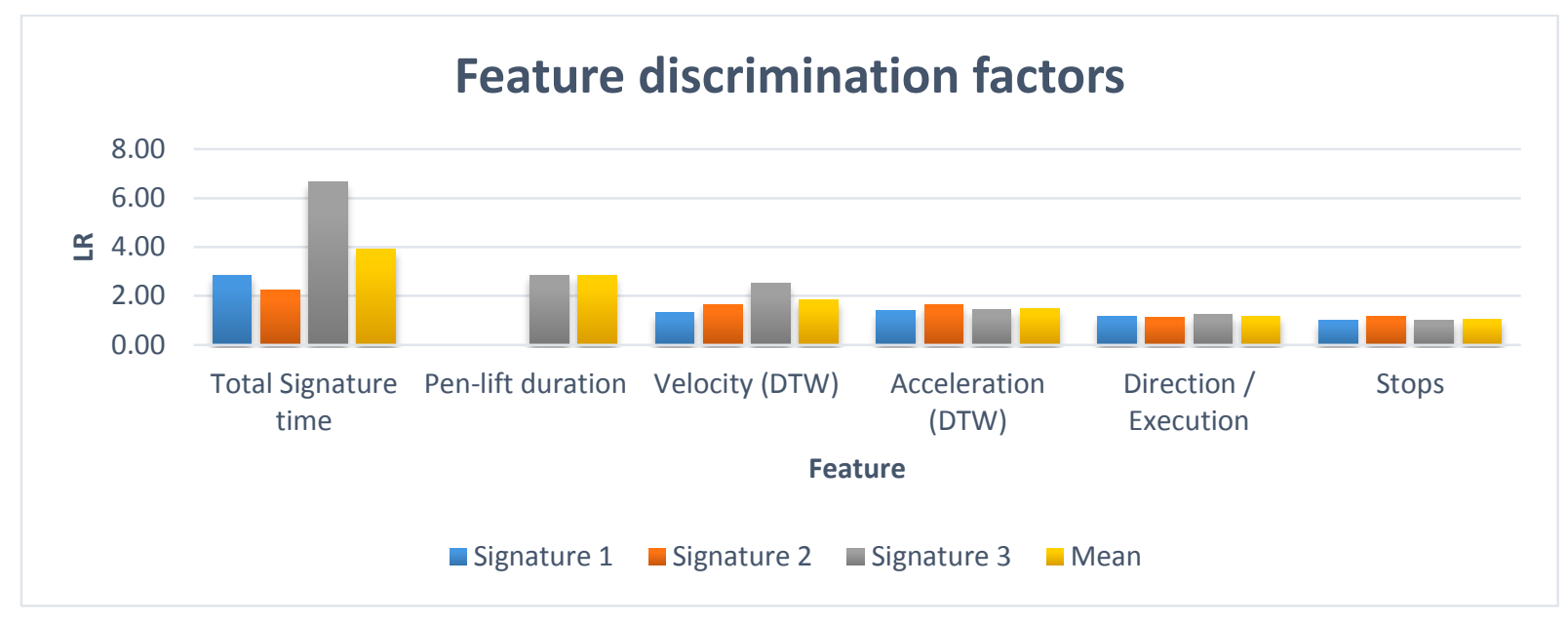

Figure 22 - Summary of feature discrimination factors for dynamic features

As Figure 21 illustrates, static features that are most often good indicators of imitation are trajectory length, bounding box dimensions, bounding box ratio, the signature internal proportions and angles (particularities), followed by shape, slope and line quality. As for dynamic features (Error! Reference source not found.), total signature time, pen-up times and velocity are the best candidates, followed by acceleration, execution and stops.

A few observations need to be made to contextualize these results. First, all of the values produced are quite low, which may well be correlated to the relatively low complexity of the signatures. Higher complexity might leave more room for error in the imitation process. This would result in higher LRs for most features, in particular for stops, total signature time and velocity. Second, one should take special care with graphical particularities' value. Some features, like starting angles and line direction, are not as discriminating as others, like letter and word internal proportions. Only stable features should be studied and used. Third, only one of the signatures was composed of multiple strokes and contained pen lifts and pen-lift durations. This feature seems very characteristic as imitators had a tendency to stop and look at the signature before continuing and so had significantly longer pen-lifts. This observation should be further explored in more complex signatures.

The generated feature utilities are only indicative of discriminatory power. They are not a means to evaluate results, otherwise the studied populations should be different in accordance to the considered hypotheses. In order to create a viable evaluation model, a probabilistic distribution of each feature should be established, along with a covariation table of features. Without those elements, the likelihood ratios per feature cannot be multiplied to generate a combined value. Feature utility may, in its current form, inform about which features to observe first and which to attribute higher value during the evaluation phase. 
On another note, an important proximity between imitations made by the same imitator was observed (Figure 23). Signatures showed consistent features between the three acquired samples for writers. A comparison using an imitator's dynamic data as reference and the other nineteen imitators as questioned data revealed that imitation data was distinguishable in sixteen cases (84.21\%) and were inside the imitator's variation in only three cases (15.79\%). Mostly, the other imitator's velocity and acceleration features were quite different from each other, as can be seen in Figure 24 .
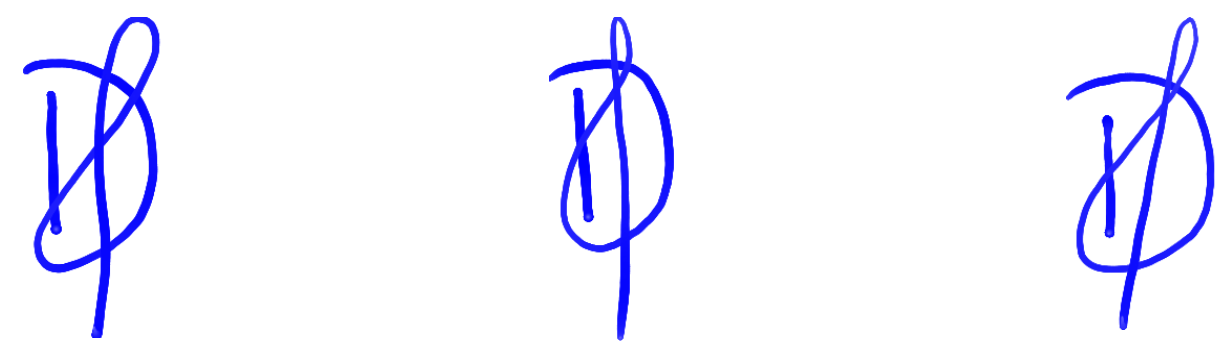

Figure 23 - Illustration of three imitated signatures by the same imitator

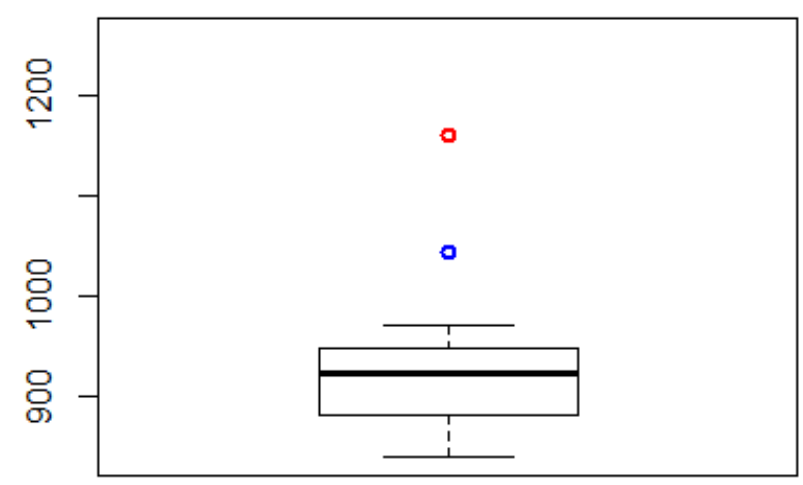

Figure 24 - Illustration of the DTW results of a set of three signatures (box) and the distances of a questioned signature with said set (the red dot corresponds to the mean distance and the blue dot corresponds to the closest distance)

A possible explanation might be that the imitator unconsciously adopts a certain "compatible" way for him to imitate the signature in question and keeps that program in mind. Thus, imitations produced by the same person are similar in graphical and dynamical features. Considering that each person has a different compatibility and motor program, the natural imitation profile is different between imitators. As such, comparisons show that imitations from the same person are closer (regarding speed and acceleration graphs) than imitations from different people. If this hypothesis is further tested and explored, it might be possible to connect imitations made by the same person.

Finally, all the feature information per signature was considered in a subjective manner in order to form a classification conclusion (genuine/imitation). None of the imitated signatures showed sufficient similarity on both dynamic and static levels to be classified as genuine. The testing procedure was not done blind, but first experiments show that combining information from the different techniques leads 
to correct conclusions. Still, dynamic information needs to be considered together with the static information. The obvious correlations between trajectory path, trajectory length, size, as well as velocity and acceleration make an independency assumption between these characteristics impossible.

\section{Conclusions}

This study focused on examining a state of the art dynamic signature system, determining whether or not the data provided was reliable and how to use the data efficiently. It further investigated whether or not within writer variation was sufficiently small to attempt to discriminate genuine and imitated signatures.

Our results show that most of the data can be relied upon, but pressure information may be problematic and was not considered. Data can be easily extracted, analyzed and compared with a few lines of computer code. In order to compare the dynamic information, dynamic time warping (DTW) was proposed and showed promising results. This automated comparison yields distances that can be used to describe feature variation and distribution, as well as being less subjective than simple qualitative approaches.

The study showed the extent of variation within writers and between genuine signatures and imitations. It also implies that separating genuine and imitated signatures is possible by studying within writer variation and considering multiple features. None of the imitations were perfect and could resist the combined consideration of both dynamic and static information, evaluated in a subjective Bayesian framework. Further research should be done into how to evaluate findings and combine features in a single coherent and logical model.

The qualification of feature utility using the likelihood ratio approach yielded interesting results, notably that the use of signature size and ratio, as well as total signing time and velocity are useful features for robust comparison. Further, this approach can be used to justify prioritizing features and assigning higher values to them in an evidence evaluation phase.

Finally, even imitators show a measure of stability in their imitations, indicating a potential means to detect imitated signatures done by the same imitator. This may enable the FDE to link cases of repeated fraud (using the same signature model) by creating probabilistic evaluation models. 


\section{Acknowledgments}

First and foremost, the authors would like to thank all of the participants in the study, especially the signers. The authors would also like to thank the many proofreaders and colleagues that were involved in the production of this paper.

\section{Bibliography}

1. Mohammed L, Found B, Caligiuri M, Rogers D. Dynamic characteristics of signatures: effects of writer style on genuine and simulated signatures. Journal of Forensic Sciences. 2015 Jan;60(1):89-94.

2. Franke K. The Influence of Physical and Biomechanical Processes on the Ink Trace Methodological foundations for the forensic analysis of signatures [Ph.D.]. Rijksuniversiteit Groningen: Rijksuniversiteit Groningen, 2005.

3. Flynn W. Executing a forensic examination of electronically captured signatures. Journal of the American Society of Questioned Document Examiners. 2012;15(1):3-10.

4. Nicolaides KA. Using acceleration plots in the forensic examination of electronically captured signatures. Journal of the American Society of Questioned Document Examiners.15(2):29-43.

5. Harralson HH. Chapter 5 - Forensic Analysis of Electronic Signatures. In: Harralson HH, editor. Developments in Handwriting and Signature Identification in the Digital Age: Anderson Publishing, Ltd.; 2013;71-111.

6. Signotec. Signotec Homepage. 2016 [updated 2016; cited 2016 20.01.2016]; Available from: http://www.signotec.com/produkte/unterschriften-pads/omega-pad/.

7. Damilic. The Autopen Company Homepage. Damilic; 2016 [updated 2016; cited 2016 20.01.2016]; Available from: http://www.damilic.com/autopen-products/office-signature-machines.

8. Team RC. R: A language and environment for statistical computing. Vienna, Austria: R Foundation for Statistical Computing; 2015.

9. Richiardi J, Ketabdar H, Drygajlo A, editors. Local and global feature selection for on-line signature verification. Document Analysis and Recognition, 2005 Proceedings Eighth International Conference on; 200529 Aug.-1 Sept. 2005. 
10. Harralson HH. Glossary. In: Harralson $\mathrm{HH}$, editor. Developments in Handwriting and Signature Identification in the Digital Age: Anderson Publishing, Ltd.; 2013;125-8.

11. Paz-Villagran V, Danna J, Velay JL. Lifts and stops in proficient and dysgraphic handwriting. Human Movement Science. 2014 Feb;33:381-94.

12. Giorgino T. Computing and Visualizing Dynamic Time Warping Alignments in R: The dtw Package. Journal of Statistical Software. 2009 2009-08-14;31(7):24.

13. Muramatsu D, Matsumoto T. Effectiveness of Pen Pressure, Azimuth, and Altitude Features for Online Signature Verification. In: Lee S-W, Li S, editors. Advances in Biometrics: Springer Berlin Heidelberg; 2007;503-12.

14. Tariq S, Sarwar S, Hussain W, editors. Classification of Features into Strong and Weak Features for an Intelligent Online Signature Verification System. First International Workshop on Automated Forensic Handwriting Analysis; 2011; Beijing, China.

15. Liwicki M. Automatic Signature Verification : In-depth investigation of novel feautures and different models. Journal of Forensic Document Examination. 2012;22:25-39.

16. Thiéry A, Marquis R, Montani I. Statistical evaluation of the influence of writing postures on on-line signatures. Study of the impact of time. Forensic Science International. 2013 Jul 10;230(13):107-16. 